\title{
UNITARITY CONSTRAINTS ON THE ELECTROWEAK THREE VECTOR BOSON VERTICES
}

\author{
U. BAUR \\ CERN - Geneva
}

and

D. ZEPPENFELD

Physics Department, University of Wisconsin Madison, WI 53706, USA

\begin{abstract}
Partial wave unitarity applied to fermion-pair annihilation into $W Z, W \gamma$ or $W^{+} W^{-}$severely limits any deviations of the $Z W W$ and $\gamma W W$ three vector boson vertices from the standard model prediction. These constraints are quantified and experimental consequences, in particular for $W$-pair production at LEP, are discussed.
\end{abstract}

CERN-TH.4892/87

November 1987 
The standard model of electroweak interactions (S.M.) has been beautifully confirmed in recent years by many experimental results. In spite of these successes there still remain crucial parts of the model that lack experimental verification, most notably the Higgs sector and the vector-boson self-interactions which are uniquely given by the gauge theory structure of the S.M. It is well known that tree unitarity e.g. for the process $e^{+} e^{-} \rightarrow W^{+} W^{-}$ uniquely restricts the three vector boson couplings $\gamma W W$ and $Z W W$ to their gauge theory values at asymptotic energies.' This leaves open, however, the possibility of anomalous contributions to the $\gamma W W$ and $Z W W$ vertices at low energies, due to novel interactions affecting the weak boson sector. Such deviations from the gauge theory prediction, if large enough, will produce observable signatures in $e^{+} e^{-} \rightarrow W^{+} W^{-2-4)}$ and in quarkantiquark annihilation into $W^{+} W^{-4,5)} W^{ \pm} \gamma^{6},{ }^{6}$ and $W^{ \pm} Z^{4,7)}$ at hadron colliders. In order to estimate the sensitivity necessary for these experiments, it is desirable to obtain upper bounds for the anomalous three vector boson couplings. Several authors have obtained such bounds by considering loop contributions to e.g. the anomalous magnetic moment of the electron or muon ${ }^{8-11)}$ or neutrino nucleon scattering. ${ }^{12)}$ However, in this approach, the three boson vertices enter at invariant masses very different from those encountered in vector boson pair production. Due to the form factor behaviour, which is implied by unitarity for any anomalous couplings, implications for vector boson pair production are ambiguous.

In this paper constraints on the most general three boson vertex that can contribute to the pair production process will be derived from partial wave unitarity of the fermionantifermion-vector boson-vector boson amplitude at arbitrary center of mass energy. Because we constrain the production amplitude for vector boson pairs, the bounds that we place on anomalous contributions to the $Z W W$ or $\gamma W W$ vertex are directly relevant for $W^{+} W^{-}, W^{ \pm} Z$, and $W^{ \pm} \gamma$ production at $e^{+} e^{-}$and/or hadron colliders, unambiguous and model-independent.

The most general $V W W$ vertex $(V=Z$ or $\gamma$ ), which can contribute to the above five vector boson pair production processes in the annihilation of effectively massless fermions, can conveniently be parametrized by the following effective Lagrangian ${ }^{2,3)}$ 


$$
\begin{aligned}
\mathcal{L}_{W W V} / g_{W W V}=i & g_{1}^{V}\left(W_{\mu \nu}^{\dagger} W^{\mu} V^{\nu}-W_{\mu}^{\dagger} V_{\nu} W^{\mu \nu}\right)+i \kappa^{V} W_{\mu}^{\dagger} W_{\nu} V^{\mu \nu} \\
& +\frac{i \lambda^{V}}{M_{W}^{2}} W_{\lambda \mu}^{\dagger} W_{\nu}^{\mu} V^{\nu \lambda}-g_{4}^{V} W_{\mu}^{\dagger} W_{\nu}\left(\partial^{\mu} V^{\nu}+\partial^{\nu} V^{\mu}\right) \\
& +g_{5}^{V} \epsilon^{\mu \nu \rho \sigma}\left(W_{\mu}^{\dagger} \vec{\partial}_{\rho} W_{\nu}\right) V_{\sigma}+i \tilde{\kappa}^{V} W_{\mu}^{\dagger} W_{\nu} \widetilde{V}^{\mu \nu} \\
& +\frac{i \tilde{\lambda}^{V}}{M_{W}^{2}} W_{\lambda \mu}^{\dagger} W^{\mu}{ }_{\nu} \widetilde{V}^{\nu \lambda}
\end{aligned}
$$

Here $W_{\mu}$ denotes the $W^{-}$field, $V^{\mu \nu}=\partial^{\mu} V^{\nu}-\partial^{\nu} V^{\mu}, A \vec{\partial}_{\rho} B \equiv A\left(\partial_{\rho} B\right)-\left(\partial_{\rho} A\right) B$, and $\tilde{V}^{\mu \nu}=\frac{1}{2} \epsilon^{\mu \nu \sigma \rho} V_{\sigma \rho}$. We choose the overall coupling constant $g_{W W V}$ as in the standard model

$$
g_{W W \gamma}=-e, \quad g_{W W Z}=-e \cot \theta_{W}
$$

In Eq. (1) $g_{4}$ violates $C P$ and $C, g_{5}$ violates $C$ and $P$ but is $C P$ even, while $\tilde{\kappa}$ and $\vec{\lambda}$ are $P$ odd and $C P$ violating. Within the S.M. they all vanish at tree level and so does $\lambda$. The only nonzero couplings within the S.M. are

$$
\begin{aligned}
& g_{1}^{V}=1 \\
& \kappa^{V}=1
\end{aligned}
$$

In order to be able to treat the production of $W^{+} W^{-}, W^{ \pm} \gamma$, and $W^{ \pm} Z$ in parallel it is convenient to introduce a vertex function $\Gamma^{\alpha \beta \mu}(q, \bar{q}, P)$ for the decay of a virtual vector boson $V_{1}$ of four momentum $P$ into two onshell vector bosons $V_{2}$ and $V_{3}$ as depicted in Fig. 1:

$$
\begin{aligned}
\Gamma^{\alpha \beta \mu}(q, \bar{q}, P)=f_{1}( & q-\bar{q})^{\mu} g^{\alpha \beta}-\frac{f_{2}}{M_{W}^{2}}(q-\bar{q})^{\mu} P^{\alpha} P^{\beta}+f_{3}\left(P^{\alpha} g^{\mu \beta}-P^{\beta} g^{\mu \alpha}\right) \\
& +i f_{4}\left(P^{\alpha} g^{\mu \beta}+P^{\beta} g^{\mu \alpha}\right)+i f_{5} \epsilon^{\mu \alpha \beta \rho}(q-\bar{q})_{\rho} \\
& -f_{6} \epsilon^{\mu \alpha \beta \rho} P_{\rho}-\frac{f_{7}}{M_{W}^{2}}(q-\bar{q})^{\mu} \epsilon^{\alpha \beta \rho \sigma} P_{\rho}(q-\bar{q})_{\sigma}
\end{aligned}
$$

The seven form factors $f_{i}$ are invariant and dimensionless functions of $q^{2}, \bar{q}^{2}$, and $P^{2}$. In Table 1 they are given in terms of the anomalous couplings of the effective Lagrangian (1) for $W^{+} W^{-}$production via $V(=\gamma, Z)$ exchange and for $W^{ \pm} V$ production via $W$ exchange.

Let us now consider the matrix element $\mathcal{M}\left(\sigma_{1} \sigma_{2}, \lambda_{2} \lambda_{3}\right)$ of the process

$$
f_{1}\left(\sigma_{1}\right) \bar{f}_{2}\left(\sigma_{2}\right) \rightarrow V_{2}\left(\lambda_{2}\right) V_{3}\left(\lambda_{3}\right)
$$

where $V_{2} V_{3}$ stands for $W^{+} W^{-}, W^{ \pm} \gamma$, or $W^{ \pm} Z$ and $\sigma_{1}, \sigma_{2}$ and $\lambda_{2}, \lambda_{3}$ denote the helicities of the particles involved. We split $\mathcal{M}$ into the S.M. contribution $\mathcal{M}^{0}$ and a piece $\Delta \mathcal{M}$, 
which is due to anomalous couplings. The interactions between vector bosons and fermions are assumed to be the same as in the S.M. where they can be described by the interaction Lagrangian

$$
\mathcal{L}_{V f_{1} f_{2}}=-e \sum_{\sigma= \pm} g_{\sigma}^{V f_{1} f_{2}} \bar{\psi}_{f_{1}} \gamma_{\mu} \frac{1}{2}\left(1+\sigma \gamma_{5}\right) \psi_{f_{2}} V^{\mu}
$$

This implies that contributions to $\Delta \mathcal{M}$ arise from $s$-channel exchange of virtual vector bosons $V$ only:

$$
\begin{gathered}
\Delta \mathcal{M}^{V}\left(\sigma_{1} \sigma_{2}, \lambda_{2} \lambda_{3}\right)=-\sqrt{2} e g_{V_{2} V_{3} V} g_{2 \sigma_{1}}^{V f_{1} f_{2}} \frac{s}{s-M_{V}^{2}} \beta \delta_{\sigma_{1},-\sigma_{2}} A_{\lambda_{2} \lambda_{3}}^{V} \\
d_{\sigma_{1}-\sigma_{2}, \lambda_{1}-\lambda_{2}}^{1}(\Theta)
\end{gathered}
$$

The dependence on the center of mass scattering angle $\Theta$ is given by the conventional $d$-functions, ${ }^{13)}$ and the dependence on the anomalous couplings is absorbed in the reduced amplitudes $A_{\lambda_{2} \lambda_{3}}$ which are given by

$$
\begin{aligned}
& A_{0 \pm}=\gamma_{2}\left[\frac{\left(f_{3}-2\right)-i f_{4} \pm \beta f_{5}}{1-\Delta} \pm \frac{i}{\beta}\left(f_{6}-i \Delta f_{5}\right)\right] \\
& A_{ \pm 0}=\gamma_{3}\left[\frac{\left(f_{3}-2\right)+i f_{4} \mp \beta f_{5}}{1+\Delta} \pm \frac{i}{\beta}\left(f_{6}-i \Delta f_{5}\right)\right] \\
& A_{ \pm \pm}=\left(f_{1}-1\right) \pm \frac{i}{\beta}\left(f_{6}-i \Delta f_{5}\right) \pm i \beta \frac{s}{M_{W}^{2}} f_{7} \\
& A_{00}=\frac{\gamma_{2} \gamma_{3}}{1-\Delta^{2}}\left[-\left(1+\beta^{2}-\Delta^{2}\right)\left(f_{1}-1\right)+\frac{s}{M_{W}^{2}} \beta^{2} f_{2}+2\left(f_{3}-2\right)\right]
\end{aligned}
$$

The abbreviations are $s=P^{2}, \Delta=\left(M_{3}^{2}-M_{2}^{2}\right) / s, \gamma_{2}=(1-\Delta) \sqrt{s} /\left(2 M_{2}\right)$, $\gamma_{3}=(1+\Delta) \sqrt{s} /\left(2 M_{3}\right)$ and

$$
\beta \sqrt{s} / 2=M_{2} \sqrt{\gamma_{2}^{2}-1}=M_{3} \sqrt{\gamma_{3}^{2} \cdots 1}
$$

Because of the various $\gamma$ and $s / M_{W}^{2}$ factors in (8) any deviation of the $f_{i}$ from their S.M. values will produce large corrections to the $f_{1} \bar{f}_{2} \rightarrow V_{2} V_{3}$ production amplitude at large center of mass energy. Since they are due to $s$-channel exchange of a vector boson which is coupled to massless fermions, they appear in the $J=1$ partial wave amplitude only. Unitarity of the $S$-matrix implies that any partial wave amplitude is bounded by a constant and this fact rules out any constant non-gauge-theory couplings.) 
In order to obtain limits for energy dependent form factors $f_{i}$, at a given center of mass energy $\sqrt{s}$, we have to analyze the unitarity constraints in detail. The partial wave expansion for the process $f_{1} \bar{f}_{2} \rightarrow V_{2} V_{3}$ is given by

$$
\mathcal{M}\left(\sigma_{1} \sigma_{2}, \lambda_{2} \lambda_{3}\right)=16 \pi \sum_{J}(2 J+1) \mathcal{T}_{\lambda_{2} \lambda_{3} ; f_{1} \sigma_{1}}^{J}(s) \delta_{\sigma_{1},-\sigma_{2}} d_{\sigma_{1}-\sigma_{2}, \lambda_{2}-\lambda_{3}}^{J}(\Theta) .
$$

The notation incorporates the fact that the fermion and the antifermion must have opposite helicities in order to annihilate, and that the discrete quantum numbers of $\bar{f}_{2}$ (like color, flavor, or generation index) are fixed by those of $f_{1}$ when Cabibbo mixing is neglected, as is appropriate for massless fermions. The $J=1$ amplitude $\mathcal{T}^{1}$ contains a (small) S.M. contribution plus a piece which can be read off from $\Delta \mathcal{M}^{V}$ in Eq. (7) and which is necessarily large when $\mathcal{T}^{J=1}$ is close to the unitarity bound. In the following we shall always neglect the S.M. contribution.

The inelastic vector boson production amplitude is related by unitarity to the amplitude $\mathcal{T}_{f_{3} \sigma_{3}, f_{1} \sigma_{1}}^{J}$ of the elastic process

$$
f_{1}\left(\sigma_{1}\right)+\bar{f}_{2}\left(\sigma_{2}\right) \longrightarrow f_{3}\left(\sigma_{3}\right)+\bar{f}_{4}\left(\sigma_{4}\right)
$$

This unitarity relation reads

$$
\begin{aligned}
& -\frac{i}{2}\left(\mathcal{T}_{f_{3} \sigma_{3} ; f_{1} \sigma_{1}}^{J}-\mathcal{T}_{f_{1} \sigma_{1} ; f_{3} \sigma_{3}}^{J *}\right)=\sum_{f \sigma} \mathcal{T}_{f \sigma ; f_{3} \sigma_{3}}^{J *} \mathcal{T}_{f \sigma ; f_{1} \sigma_{1}}^{J} \\
& \quad+\beta \sum_{\lambda_{2} \lambda_{3}} \mathcal{T}_{\lambda_{2} \lambda_{3} ; f_{3} \sigma_{3}}^{J *} \mathcal{T}_{\lambda_{2} \lambda_{3} ; f_{1} \sigma_{1}}^{J}+\sum_{n} \mathcal{T}_{n ; f_{3} \sigma_{3}}^{J *} \mathcal{T}_{n ; f_{1} \sigma_{1}}^{J}
\end{aligned}
$$

with $\beta$ being defined in Eq. (9). The last term sums all inelastic intermediate states $|n\rangle$ which are not electroweak vector boson pairs. Instead of studying the scattering of a fixed state $\left|f_{1} \bar{f}_{2}\right\rangle$, e.g. a $u \bar{d}$ pair with a fixed color, it is more convenient to use arbitrary linear combinations $|x\rangle=\sum_{f_{1} \sigma_{1}} x_{f_{1} \sigma_{1}}\left|f_{1}\left(\sigma_{1}\right) \bar{f}_{2}\left(-\sigma_{1}\right)\right\rangle$, i.e. to contract Eq. (12) with $x_{f_{3} \sigma_{3}}^{*} x_{f_{1} \sigma_{1}}$. This results in the unitarity relation

$$
\operatorname{Im} \mathcal{T}_{x ; x}^{J}=\frac{1}{\left\|x^{2}\right\|}\left|\mathcal{T}_{x ; x}^{J}\right|^{2}+\beta \sum_{\lambda_{2} \lambda_{3}}\left|\mathcal{T}_{\lambda_{2} \lambda_{3} ; x}^{J}\right|^{2}+\sum_{n}\left|\mathcal{T}_{n ; x}^{J}\right|^{2}
$$

where $\|x\|^{2}=\sum_{f \sigma}\left|x_{f \sigma}\right|^{2}$ and the $x$ indices mean $e . g$.

$$
\mathcal{T}_{\lambda_{2} \lambda_{3} ; x}^{J}=\sum_{f \sigma} x_{f \sigma} \mathcal{T}_{\lambda_{2} \lambda_{3} ; f \sigma}^{J}
$$

Equation (13) is a quadratic equation for the positive imaginary part of $\mathcal{T}_{x ; x}^{J}$ which is 
consistent only if

$$
\beta \sum_{\lambda_{2} \lambda_{3}} \frac{\left|\mathcal{T}_{\lambda_{2} \lambda_{3} ; x}^{J}\right|^{2}}{\|x\|^{2}} \leq \frac{1}{4}
$$

Combining this limit with Eqs. (10) and (7) for the $J=1$ partial wave amplitude we obtain the desired unitarity bounds for the reduced amplitudes $A_{\lambda_{2} \lambda_{3}}^{V}$ :

$$
\sum_{\lambda_{2} \lambda_{3}}\left|\sum_{V} \frac{g_{V_{2} V_{3} V}}{-e}\left(\sum_{f_{1} \sigma_{1}} g_{2 \sigma_{1}}^{V f_{1} f_{2}} \frac{x_{f_{1} \sigma_{1}}}{\|x\|}\right) \frac{s}{s-M_{V}^{2}} A_{\lambda_{2} \lambda_{3}}^{V}\right|^{2} \leq \frac{18}{\alpha^{2} \beta^{3}} .
$$

Here $\sum_{V}$ indicates that in $W^{+} W^{-}$production both photon- and $Z$-exchange must be taken into account while in $W^{ \pm} Z$ or $W^{ \pm} \gamma$ production the only contribution is from $s$-channel $W$ exchange. $\alpha$ is the QED fine structure constant.

The linear combination $|x\rangle$ of fermion antifermion states has to be chosen such as to maximize the coefficient of $A_{\lambda_{2} \lambda_{3}}^{V}$ in Eq. (16). For $W^{ \pm} Z$ or $W^{ \pm} \gamma$ production this is a trivial task since the charged current couplings are universal: simply choose

$$
|x\rangle=\left|\sum_{\text {colors }}(u \bar{d}+c \bar{s}+t \bar{b})+\left(\nu_{e} e^{+}+\nu_{\mu} \mu^{+}+\nu_{\tau} \tau^{+}\right)\right\rangle
$$

or the charge conjugate state, i.e. $x_{f_{1} \sigma_{1}} /\|x\|=1 / \sqrt{12}$. The resultant unitarity bounds are

$$
\left\{\sum_{\lambda_{2} \lambda_{3}}\left|A_{\lambda_{3} \lambda_{3}}\right|^{2}\right\}^{1 / 2} \leq \frac{\sqrt{3}}{\alpha \beta^{3 / 2}} \frac{\sin ^{2} \theta_{W}}{\cos \theta_{W}}\left(1-\frac{M_{W}^{2}}{s}\right)=K_{17}
$$

for $W^{ \pm} Z$ production, and

$$
\left\{\sum_{\lambda_{2} \lambda_{3}}\left|A_{\lambda_{2} \lambda_{3}}\right|^{2}\right\}^{1 / 2} \leq \frac{1}{\beta^{1 / 2}} \frac{\sqrt{3}}{\alpha} \sin \theta_{W}=K_{18}
$$

for $W^{ \pm} \gamma$ production. For $W^{+} W^{-}$production the optimal choices of $|x\rangle$ lead to the bounds

$$
\left\{\sum_{\lambda_{2} \lambda_{3}}\left|A_{\lambda_{2} \lambda_{3}}^{\gamma}\right|^{2}\right\}^{1 / 2} \leq \frac{1}{\alpha \beta^{3 / 2}}\left\{\frac{3}{5}\left(3-6 \sin ^{2} \theta_{W}+8 \sin ^{4} \theta_{W}\right)\right\}^{1 / 2}=K_{19}
$$

and

$$
\left\{\sum_{\lambda_{2} \lambda_{3}}\left|A_{\lambda_{2} \lambda_{3}}^{Z}\right|^{2}\right\}^{1 / 2} \leq \frac{1}{\alpha \beta^{3 / 2}} \sqrt{\frac{3}{10}} 4 \sin ^{2} \theta_{W}\left(1-\frac{M_{Z}^{2}}{s}\right)=K_{20}
$$

for the photon- and $Z$-exchange amplitudes. Equations (17)-(20) are the desired constant 
unitarity bounds which tell us that any deviation $\Delta g_{1}, \Delta \kappa, \lambda$, etc. from the S.M. three boson vertices must actually be a form factor which vanishes at asymptotically large energies.

A slight complication arises because the three basic processes $W^{+} W^{-}$production via virtual $V$-exchange, $W^{+} V$ - and $W^{-} V$-production probe these form factors at different invariant masses. In the $W^{-} W^{+} V$ vertex function (with all three vector bosons outgoing) let us call the $W^{-}, W^{+}$and $V$ invariant masses $q^{2}, \bar{q}^{2}$, and $P^{2}$, respectively. Then the anomalous couplings (form factors) $a\left(q^{2}, \bar{q}^{2}, P^{2}\right)$ which are probed are $a\left(M_{W}^{2}, M_{W}^{2}, s\right)$ in $W^{+} W^{-}$production, and $a\left(s, M_{W}^{2}, M_{V}^{2}\right)$ and $a\left(M_{W}^{2}, s, M_{V}^{2}\right)$ in $W^{+} V$ and $W^{-} V$ production. Fortunately the latter two are related by CPT,

$$
a\left(q^{2}, \bar{q}^{2}, P^{2}\right)=a\left(\bar{q}^{2}, q^{2}, P^{2}\right)^{*}
$$

and bounds for the first two processes are sufficient.

It is easy now to turn the inequalities (17)-(20) into bounds for the anomalous couplings in the effective Lagrangian (1). In Table 2 we have summarized these bounds assuming that only one anomalous coupling at a time is different from zero. The expressions are valid at arbitrary center of mass energy $\sqrt{s}$, except for the $W Z$ case, where corrections of order $M_{W}^{2} / s$ and $M_{Z}^{2} / s$ have been neglected in order to avoid lengthy expressions. For $W^{ \pm} \gamma$ production zero bounds are given for $\left|\Delta g_{1}^{\gamma}\right|,\left|g_{4}^{\gamma}\right|$, and $\left|g_{5}^{\gamma}\right|$ : electromagnetic gauge invariance forbids these couplings for onshell photons (for a discussion see Ref. 3 ).

The bounds in Table 2 assume that no cancellations occur between different anomalous couplings. Relaxing this assumption one finds that large cancellations are indeed possible close to threshold, where the unitarity bounds are rather weak anyway. For $s \gg M_{Z}^{2}, M_{W}^{2}$, the rigorous bounds and the bounds of Table 2 merge, however. Typically, at $\sqrt{s}=1 \mathrm{TeV}$, the rigorous bounds are only $3-5 \%$ weaker than the ones presented in the table. There is an exception to this statement, namely $\Delta g_{1}^{Z}$ and $g_{4}^{Z}$ for $W^{+} Z$ production. At $s \gg M_{Z}^{2}$ they produce the largest effect in

$$
\left|A_{00}\right|^{2} \approx\left(\frac{s}{2 M_{W} M_{Z}}\right)^{2}\left|\Delta g_{1}-i g_{4}\right|^{2},
$$

while $\Delta g_{1}$ alone receives its strongest bound from

$$
\left|A_{+0}\right|^{2}+\left|A_{-0}\right|^{2} \approx \frac{2 s}{M_{Z}^{2}}\left|\Delta g_{1}\right|^{2}+\ldots .
$$

For $\Delta g_{1} \approx i g_{4}$ the asymptotic bound for $\Delta g_{1}$ and $g_{4}$ is thus reduced by one power of $\sqrt{s}$. However, this particular cancellation between $\Delta g_{1}$ and $g_{4}$ requires a $90^{\circ}$ phase shift 
between the two, which in general is only expected close to a resonance in the $W Z$ cross section. Off resonance the asymptotic bound implied by Table 2 should be valid. A similar cancellation is possible between $g_{5}$ and $i \bar{\kappa}$ in $W Z$ production. In this case, however, the rigorous bound retains the power of $s$ given in Table 2 .

The bounds presented so far have very little interest for low energy experiments and even for $W^{+} W^{-}$production at LEP at $\sqrt{s} \approx 200 \mathrm{GeV}$ when no assumption on the form factor behavior is made. For deviations of the $Z W W / \gamma W W$ couplings from the gauge theory value, which are produced by some novel interactions operative at a scale $\Lambda$, one should expect, however, that they stay essentially constant for $\sqrt{s}<\Lambda$ and start decreasing only when the scale $\Lambda$ is reached or surpassed, very much like the well-known nucleon form factors. The precise $s$-dependence is unknown, of course, so we will make the simplest possible ansatz, assuming a constant anomalous coupling for $\sqrt{s}<\Lambda$ which drops abruptly to zero at $\sqrt{s}=\Lambda$. This has the advantage that the bounds of Table 2 can immediately be reinterpreted as a relation between the largest permissible low energy value of the form factor and the scale of new physics $\Lambda$ : simply replace $\sqrt{s}$ by $\Lambda$ in the table.

It is reasonable, of course, to assume the same cut-off scale $\Lambda$ in $W^{+} W^{-}$production as in $W^{ \pm} Z / \gamma$ production and hence we use the ansatz $(\theta(x)$ is the step function)

$$
a\left(q^{2}, \bar{q}^{2}, P^{2}\right)=a^{0} \theta\left(\Lambda^{2}-q^{2}\right) \theta\left(\Lambda^{2}-\bar{q}^{2}\right) \theta\left(\Lambda^{2}-P^{2}\right)
$$

for any of the anomalous couplings $a=\Delta g_{1}, \Delta \kappa, \lambda$, etc., which combines the bounds derived from the various vector boson final states. When $\Lambda$ is measured in $\mathrm{TeV}$ and for $M_{W}=81.2 \mathrm{GeV}, M_{Z}=92.5 \mathrm{GeV}, \sin ^{2} \theta_{W}=1-M_{W}^{2} / M_{Z}^{2} \approx 0.23$ and $\alpha=\alpha\left(M_{W}\right) \approx$ $1 / 127.5$ we find for $\Lambda \gg M_{Z}$ :

$$
\begin{array}{rc}
\left|\Delta g_{1}^{Z}\right|<0.87 / \Lambda^{2} & \\
\left|\Delta \kappa^{Z}\right|<0.85 / \Lambda^{2} & \left|\Delta \kappa^{\gamma}\right|<1.86 / \Lambda^{2} \\
\left|\lambda^{Z}\right|<0.54 / \Lambda^{2} & \left|\lambda^{\gamma}\right|<0.99 / \Lambda^{2} \\
\left|g_{4}^{Z}\right|<0.87 / \Lambda^{2} & \\
\left|g_{5}^{Z}\right|<3.3 / \Lambda & \\
\left|\tilde{\kappa}^{Z}\right|<5.2 / \Lambda & \left|\tilde{\kappa}^{\gamma}\right|<11.5 / \Lambda \\
\left|\tilde{\lambda}^{Z}\right|<0.54 / \Lambda^{2} & \left|\tilde{\lambda}^{\gamma}\right|<0.99 / \Lambda^{2}
\end{array}
$$

No low energy bound is given for $\Delta g_{1}^{\gamma}, g_{4}^{\gamma}, g_{5}^{\gamma}$ because they have to vanish at $P^{2}=0$ due to gauge invariance. At this point we want to stress once more that the bounds of 
Eq. (23) are valid only for $\Lambda \gtrsim 1 \mathrm{TeV}$. At $\Lambda=1 \mathrm{TeV}$ the rigorous bounds are weaker by $5 \%$ at most, but this error increases to $20 \%$ at $\Lambda=500 \mathrm{GeV}$ and one may want to return to Eqs. (17)-(20) to obtain more accurate limits for even smaller cut-off scales.

The attentive reader has probably noticed that the bounds for $\lambda, \Delta g_{1}$, and $\Delta \kappa$ all decrease like $1 / \Lambda^{2}$ even though $\lambda$ multiplies a dimension 6 operator in the effective Lagrangian (2.1) while $g_{1}$ and $\kappa$ are the coefficients of dimension 4 operators only. The reason is that longitudinal polarization states essentially do not couple via the $\lambda$ term in (2.1) because the leading term in the longitudinal polarization vector $\epsilon_{\mu}=q_{\mu} / M+\mathcal{O}\left(M / q^{0}\right)$ vanishes when inserted into the field strength $W_{\mu \nu}$ or $V_{\mu \nu}$. This suppression is not operative in the $g_{1}$ and $\kappa$ terms which hence are enhanced in helicity 0 amplitudes. We thus find that there is no reason why the particular dimension 6 operators in (2.1) should be less important than anomalous dimension 4 operators, e.g. when effects of anomalous couplings on vector boson pair production close to threshold are considered. Truncating the effective Lagrangian at the dimension 4 level is not justified.

One may wonder whether elastic unitarity applied to vector boson scattering would yield much more stringent bounds on anomalous three boson couplings since the weak fermion boson coupling is replaced by a strong three vector boson vertex. For a very restricted set of anomalous three- and four-boson vertices with dimension 4 this question has been analyzed in Ref. 9,14. Suzuki finds bounds for $\Delta \kappa^{\gamma}$ and $\Delta \kappa^{Z}$ which are more stringent than those given in Eq. (23) by a factor 2.3 at most. This surprising result can at least partially be explained by the large degeneracy of contributing fermion-antifermion states in the unitarity relation (12), which is not present in vector boson elastic scattering. Also, as demonstrated by Bilchak et al., ${ }^{14)}$ large cancellations between three- and fourvector boson-couplings occur in the latter process. It is not clear at present whether any useful bounds on three boson couplings remain, when higher dimensional four boson operators are included, which are no longer suppressed close to the unitarity limit.

The large number $N$ of fermion pair states contributing to the unitarity relation (12) implies that the bounds that we have derived are better than tree level unitarity. Any loop corrections are down by powers of $1 / \sqrt{N}$ and hence suppressed. To the extent that the $J=1$ component of the $f_{1} \bar{f}_{2} \rightarrow V_{2} V_{3}$ scattering amplitude is used to define the $V_{1} V_{2} V_{3}$ vertex function, the bounds of Table 2 are even exact (modulo neglect of small S.M. contributions).

The bounds of Eq. (23) may be compared to the results of calculations taking into account loop effects of anomalous three vector boson vertices on low energy quantities 
like $(g-2)_{\mu}{ }^{8-11)}$ low energy neutrino scattering, ${ }^{12)}$ or on the $W-Z$ mass difference. ${ }^{9,15)}$ Usually anomalous values of $\kappa$ and $\lambda$ only are assumed in these calculations, and the bounds are up to a factor 10 better for $\kappa^{\gamma}$ and $\lambda^{\gamma}$, and a factor $65(5)$ better for $\kappa^{Z}\left(\lambda^{Z}\right)$. The bounds on the $C$ or $P$ violating $Z W W$ couplings are the best available to our knowledge. The most stringent of the low energy bounds can actually be traced to quadratically divergent (i.e. proportional to $\Lambda^{2}$ ) contributions to the $W$ and $Z$ vacuum polarization. Since the smallness of the $W$ and $Z$ mass (compared to the cut-off scale $\Lambda$ ) and hence the structure of the vacuum polarization is rather ill understood in non-gauge models, these bounds should be taken with a grain of salt. Sticking e.g. to $g-2$ bounds, where these ambiguities do not arise, one finds low energy bounds for $\kappa^{\gamma}$ or $\lambda^{\gamma}$ which are comparable to the unitarity bounds of Eq. (23) at $\Lambda \approx 1 \mathrm{TeV}$.

The unitarity bounds have important implications for the sensitivity requirements of future accelerators. Assuming some novel strong interactions in the electroweak vector boson sector at a scale $\Lambda$, deviations in the $Z W W$ or $\gamma W W$ vertex are to be expected somewhat below the bounds of Eq. (23), at least for $g_{1}, \kappa$, and $\lambda$ which do not violate any discrete symmetries. At LEP, in $W^{+} W^{-}$production, these three couplings are measurable with an accuracy of $\approx \pm 0.1^{16)}$ which means that a significant signal for new physics can be observed only if $\Lambda \lesssim 2-3 \mathrm{TeV}$. If $\Lambda$ is larger, one may have to wait for the LHC or SSC where deviations of order $10^{-2}$ can give clear signals ${ }^{7)}$ which implies that $\Lambda \lesssim 10-20 \mathrm{TeV}$ can be probed. In order to probe the TeV scale, one clearly must be sensitive to anomalous couplings smaller than $0.5 \mathrm{in}$ any experiment.

\section{ACKNOWLEDGEMENTS}

This research was supported in part by the University of Wisconsin Research Committee with funds granted by the Wisconsin Alumni Research Foundation, and in part by the U. S. Department of Energy under contract DE-AC:02-76ER00881. 


\section{REFERENCES}

1. J. M. Cornwall, D. N. Levin and G. Tiktopoulos, Phys. Rev. Lett. 30, 1268 (1973); Phys. Rev. D10, 1145 (1974);

C. H. Llewellyn Smith, Phys. Lett. 46B, 233 (1973);

S. D. Joglekar, Ann. Phys. 83, 427 (1974).

2. K. Gaemers and G. Gounaris, Z. Phys. C1, 259 (1979).

3. K. Hagiwara, et al., Nucl. Phys. B282, 253 (1987).

4. M. Kuroda, et al., Nucl. Phys. B284, 271 (1987).

5. C.-H. Chang and S.-C. Lee, FERMILAB-PUB-87/64-T and 87/68-T (1987);

J. Stroughair and C. Bilchak, Z. Phys. C23, 377 (1984).

6. J. Cortés, K. Hagiwara, and F. Herzog, Nucl. Phys. B278, 26 (1986).

7. D. Zeppenfeld and S. Willenbrock, Madison preprint MAD/PH/352 (1987).

8. F. Herzog, Phys. Lett. 148B, 355 (1984).

9. M. Suzuki, Phys. Lett. 153B, 289 (1985).

10. J.C. Wallet, Phys. Rev. D32, 813 (1985).

11. A. Grau and J. A. Grifols, Phys. Lett. 154B, 283 (1985).

12. J.A. Grifols, S. Peris and J. Solà, Phys. Lett. 197B, 437 (1987).

13. Particle Data Group, M. Aguilar-Benitez et al., Phys. Lett. 170B, 1 (1986).

14. C. Bilchak, M. Kuroda, and D. Schildknecht, CERN-TH.4823/87 (1987).

15. H. Neufeld, D. Schildknecht, and J.D. Stroughair, CERN-TH.4720/87 (1987);

J. A. Grifols, S. Peris, and J. Solà, DESY preprint DESY-86-055 (1986);

J.J. van der Bij, Phys. Rev. D 35, 1088 (1987).

16. G. Barbiellini et al, in Physics at LEP, ed. J. Ellis and R. D. Peccei, CERN report 86-02, vol. 2, p. 1 (1986);

D. Treille et al., in Proceedings of the ECFA workshop on LEP 200, Aachen (1986), ed. A. Boehm and W. Hoogland, CERN report 87-08, vol. 2 (1987). 


\section{TABLE 1}

Form factors $f_{i}$ to be used in the vertex function $\Gamma^{\alpha \beta \mu}$ for $V^{*} \rightarrow W^{+} W^{-}$and $\left(W^{ \pm}\right)^{*} \rightarrow$ $W^{ \pm} V$ (from Refs. 3, 7 ).

\begin{tabular}{ccc} 
& $V^{*} \rightarrow W^{+} W^{-}$ & $\left(W^{ \pm}\right)^{*} \rightarrow W^{ \pm} V$ \\
\hline$f_{1}$ & $g_{1}^{V}+\frac{P^{2}}{2 M_{W}^{2}} \lambda^{V}$ & $\mp \frac{1}{2}\left(g_{1}^{V}+\kappa^{V} \pm i g_{4}^{V}+\frac{P^{2}}{M_{W}^{2}} \lambda^{V}\right)$ \\
$f_{2}$ & $\lambda_{V}$ & $\mp \lambda_{V}$ \\
$f_{3}+i f_{4}$ & $g_{1}^{V}+\kappa^{V}+\lambda^{V}+i g_{4}^{V}$ & $\mp\left(2 g_{1}^{V}+\frac{M_{V}^{2}}{M_{W}^{2}} \lambda^{V}\right)$ \\
$f_{3}-i f_{4}$ & $g_{1}^{V}+\kappa^{V}+\lambda^{V}-i g_{4}^{V}$ & $\mp\left(g_{1}^{V}+\kappa^{V}+\lambda^{V}\right)+i g_{4}^{V}$ \\
$f_{6}-i f_{5}$ & $\tilde{\kappa}^{V}-\tilde{\lambda}^{V}-i g_{5}^{V}$ & $\pm\left(\bar{\kappa}^{V}+\tilde{\lambda}^{V}\right)-i g_{5}^{V}$ \\
$f_{6}+i f_{5}$ & $\tilde{\kappa}^{V}-\tilde{\lambda}^{V}+i g_{5}^{V}$ & $\pm \frac{M_{V}^{2}}{M_{W}^{2}} \tilde{\lambda}^{V}-2 i g_{5}^{V}$ \\
$f_{7}$ & $-\frac{1}{2} \tilde{\lambda}^{V}$ & $\pm \frac{1}{2} \tilde{\lambda}^{V}$
\end{tabular}


Unitarity bounds on the form factors corresponding to the anomalous couplings in the effective Lagrangian (1). Only one form factor is assumed to differ from zero at a time. Results for $W^{ \pm} Z$ production are valid at $s \gg M_{W}^{2}, M_{Z}^{2}$ only. $K_{17}, \ldots, K_{20}$ are given in Eqs. (17)-(20).

\begin{tabular}{|c|c|c|c|}
\hline process & $W^{+} W^{-}$ & $W^{ \pm} \gamma$ & $W^{ \pm} Z$ \\
\hline$\gamma$ & $\frac{\sqrt{s}}{2 M_{W}}$ & $\frac{\sqrt{s}}{M_{W}}$ & - \\
\hline$\beta$ & $\left(1-4 \frac{M_{W}^{2}}{s}\right)^{1 / 2}$ & $1-\frac{M_{W}^{2}}{s}$ & - \\
\hline$K$ & $K_{19}(V=\gamma), K_{20}(V=Z)$ & $K_{18}$ & $K_{17}$ \\
\hline$\left|\Delta g_{1}^{V}\right|$ & $K / \sqrt{4 \gamma^{2}+3}$ & 0 & $2 K \frac{M_{W} M_{Z}}{s}$ \\
\hline$\left|\Delta \kappa^{V}\right|$ & $K /\left(2 \gamma \sqrt{\gamma^{2}+1}\right)$ & $\sqrt{2} K / \sqrt{\gamma^{2}+1}$ & $\sqrt{2} K \frac{M_{W}}{\sqrt{s}}$ \\
\hline$\left|\lambda^{V}\right|$ & $K /\left(2 \gamma \sqrt{2 \gamma^{2}+1}\right)$ & $\sqrt{2} K /\left(\gamma \sqrt{\gamma^{2}+1}\right)$ & $\sqrt{2} K \frac{M_{W}^{2}}{s}$ \\
\hline$\left|g_{4}^{V}\right|$ & $K /(2 \gamma)$ & 0 & $2 K \frac{M_{W} M_{z}}{s}$ \\
\hline$\left|g_{5}^{V}\right|$ & $K /\left(2 \sqrt{\gamma^{2}-1}\right)$ & 0 & $K \sqrt{\frac{2}{s}} \frac{M_{Z} M_{W}}{\sqrt{M_{Z}^{2}+4 M_{W}^{2}}}$ \\
\hline$\left|\bar{\kappa}^{V}\right|$ & $K \beta / \sqrt{4 \gamma^{2}+2}$ & $\sqrt{2} K / \sqrt{\gamma^{2}+1}$ & $\sqrt{2} K \frac{M_{W}}{\sqrt{s}}$ \\
\hline$\left|\bar{\lambda}^{V}\right|$ & $K \beta / \sqrt{8 \gamma^{4}-4 \gamma^{2}+2}$ & $\sqrt{2} K /\left(\gamma \sqrt{\gamma^{2}+1}\right)$ & $\sqrt{2} K \frac{M_{W}^{2}}{s}$ \\
\hline
\end{tabular}




\section{FIGURE CAPTION}

1. Feynman rule for the general $V_{1} V_{2} V_{3}$ vertex. The vertex function $\Gamma$ is given in Eq. (4).

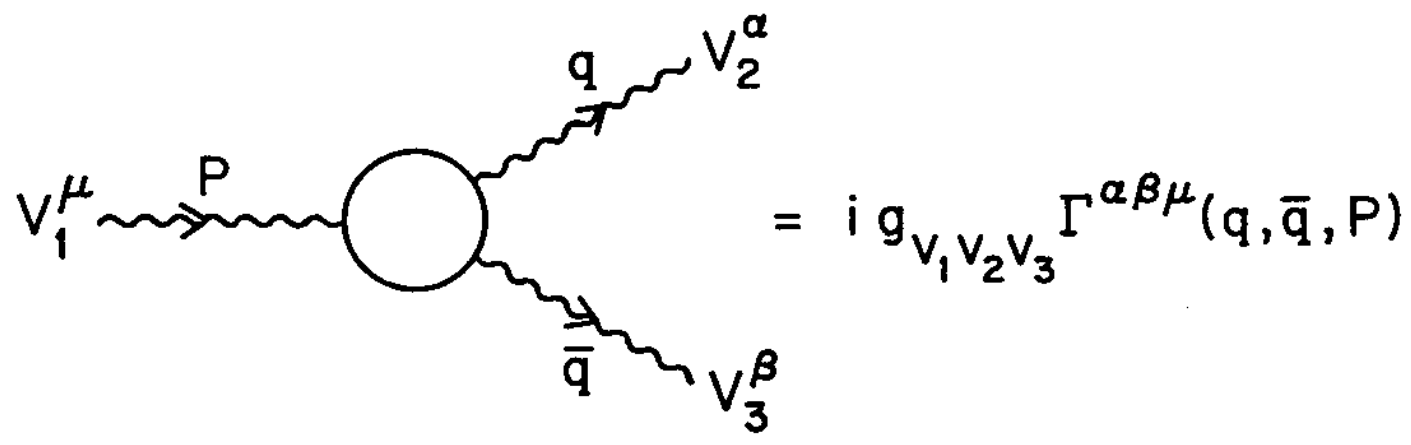

Fig. 1 